\title{
Effects of anisotropy and void shape on cavitation instabilities
}

\section{Tvergaard, Viggo; Legarth, Brian Nyvang}

\section{Published in:}

International Journal of Mechanical Sciences

Link to article, DOI:

10.1016/j.ijmecsci.2018.12.014

Publication date:

2019

Document Version

Peer reviewed version

Link back to DTU Orbit

Citation (APA):

Tvergaard, V., \& Legarth, B. N. (2019). Effects of anisotropy and void shape on cavitation instabilities. International Journal of Mechanical Sciences, 152, 81-87. https://doi.org/10.1016/j.ijmecsci.2018.12.014

\section{General rights}

Copyright and moral rights for the publications made accessible in the public portal are retained by the authors and/or other copyright owners and it is a condition of accessing publications that users recognise and abide by the legal requirements associated with these rights.

- Users may download and print one copy of any publication from the public portal for the purpose of private study or research.

- You may not further distribute the material or use it for any profit-making activity or commercial gain

- You may freely distribute the URL identifying the publication in the public portal

If you believe that this document breaches copyright please contact us providing details, and we will remove access to the work immediately and investigate your claim 


\section{Accepted Manuscript}

Effects of anisotropy and void shape on cavitation instabilities

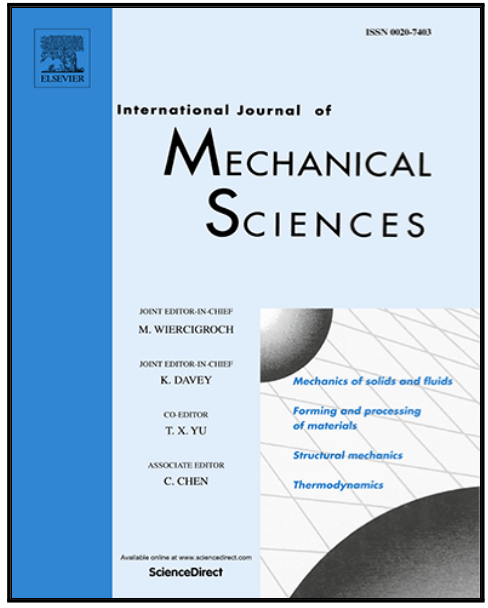

To appear in:

International Journal of Mechanical Sciences

Received date: $\quad 4$ September 2018

Accepted date: 10 December 2018

Please cite this article as: Viggo Tvergaard, Brian Nyvang Legarth, Effects of anisotropy and void shape on cavitation instabilities, International Journal of Mechanical Sciences (2018), doi: https://doi.org/10.1016/j.ijmecsci.2018.12.014

This is a PDF file of an unedited manuscript that has been accepted for publication. As a service to our customers we are providing this early version of the manuscript. The manuscript will undergo copyediting, typesetting, and review of the resulting proof before it is published in its final form. Please note that during the production process errors may be discovered which could affect the content, and all legal disclaimers that apply to the journal pertain. 


\section{Highlights}

- Nonlinear 3D finite element computations carried out for cell models

- Anisotropic plasticity is modelled in the analyses

- Cavitation instabilities are studied in the full 3D solution

- There is focus on void shape evolution

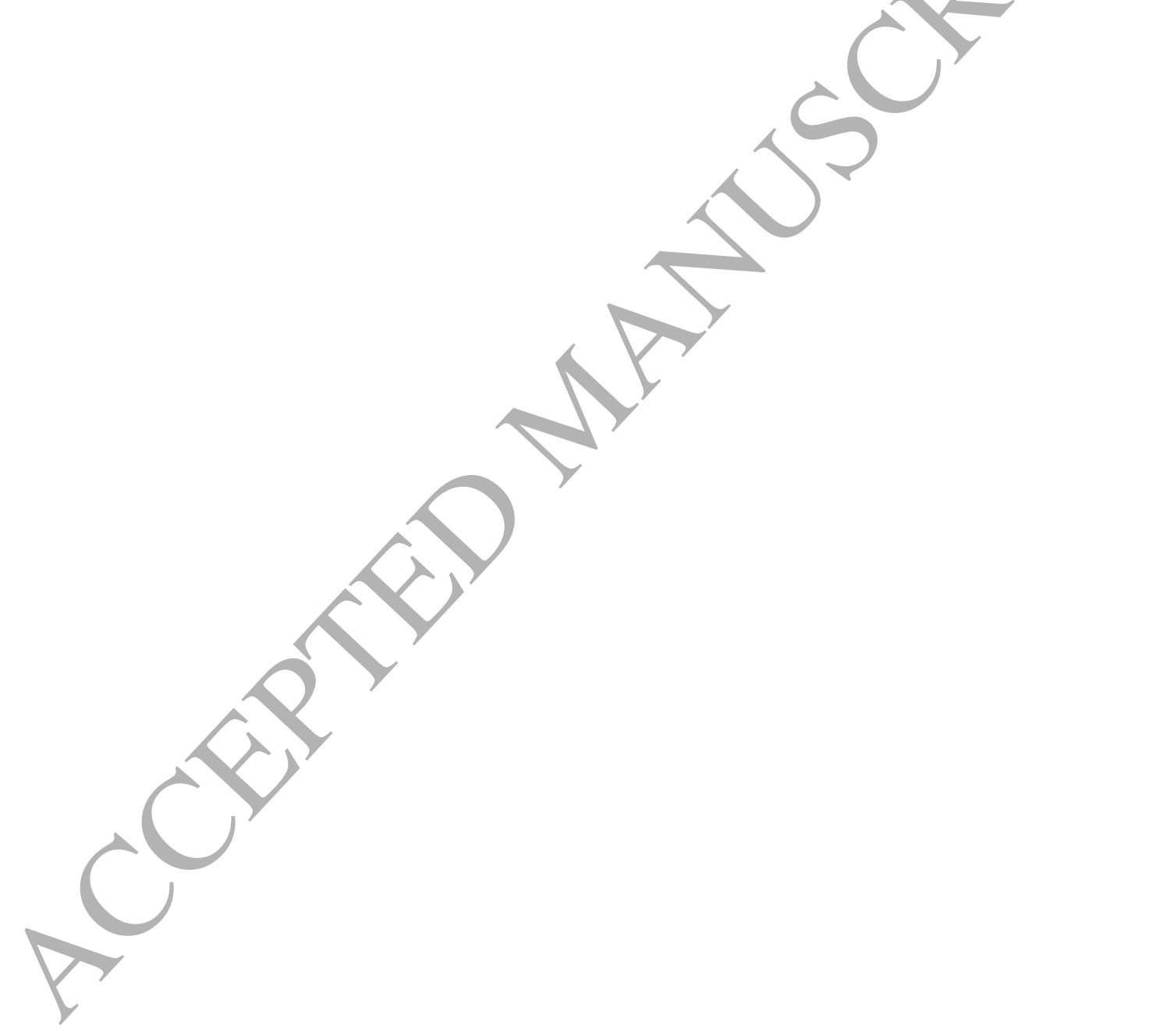




\section{Graphical Abstract}

Title

Effects of anisotropy and void shape on cavitation instabilities

authored by

Viggo Tvergaard and Brian Nyvang Legarth*

Department of Mechanical Engineering, Solid Mechanics

Technical university of Denmark

DK-2800 Kgs. Lyngby, Denmark

*Corresponding author: bnl@mek.dtu.dk

The influence of plastic anisotropy on cavitation instabilities is studied by analyzing full three

dimensional cell models containing a small void. The anisotropic material is represented by an elastic-

viscoplastic material with a small rate hardening exponent, and different 3D stress states are considered

in the range of high stress triaxialities where the occurrence of cavitation instabilities can be expected.

For isotropic plasticity the final shape of the growing voids is close to spherical, whether or not the

initial shape was spherical. But for anisotropic materials the final void shape develops towards a

spheroidal shape, which is characteristic for the anisotropy considered. This is studied both for initially

spherical voids and for initially spheroidal voids. The critical stress levels found depend much on the

anisotropy, but shows little influence of the initial void shape considered.
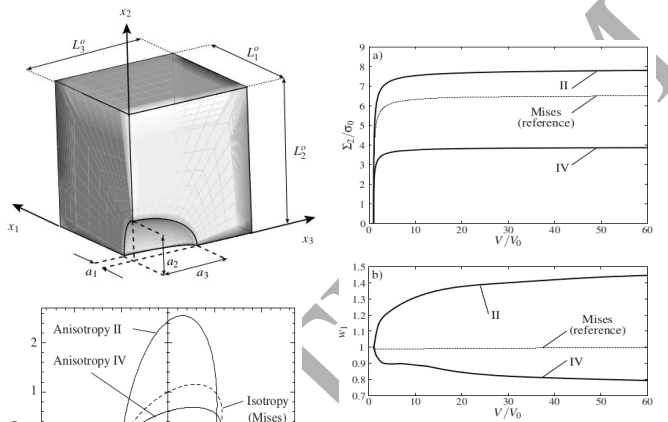$$
\text { : }
$$

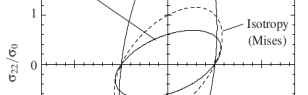

:
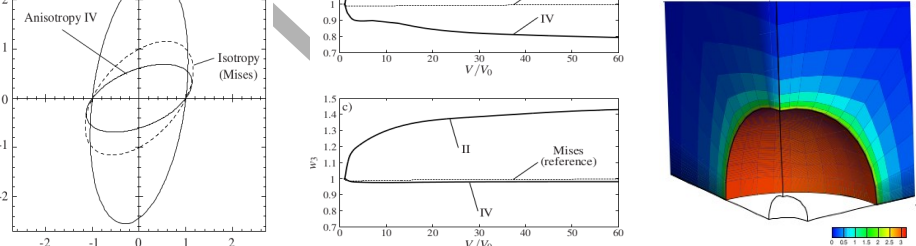

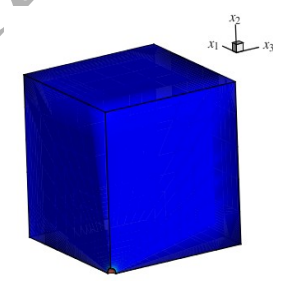

हो:

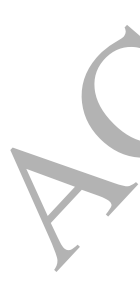




\title{
Effects of anisotropy and void shape on cavitation instabilities
}

\author{
Viggo Tvergaard and Brian Nyvang Legarth* \\ Department of Mechanical Engineering, Solid Mechanics, Technical university of Denmark \\ DK-2800 Kgs. Lyngby, Denmark \\ *Corresponding author: bnl@mek.dtu.dk
}

\begin{abstract}
The influence of plastic anisotropy on cavitation instabilities is studied by analyzing full three dimensional cell models containing a small void. The anisotropic material is represented by an elastic-viscoplastic material with a small rate hardening exponent, and different 3D stress states are considered in the range of high stress triaxialities where the occurrence of cavitation instabilities can be expected. For isotropic plasticity the final shape of the growing voids is close to spherical, whether or not the initial shape was spherical. But for anisotropic materials the final void shape develops towards a spheroidal shape, which is characteristic for the anisotropy considered. This is studied both for initially spherical voids and for initially spheroidal voids. The critical stress levels found depend much on the anisotropy, but shows little influence of the initial void shape considered.
\end{abstract}

Keywords: Anisotropy, large strain viscoplasticity, void shape, cavitation instability.

\section{Introduction}

Cavitation instabilities have been found for a single void in an infinite elastic-plastic solid, in cases where the stress level is high enough, so that the work released in the fields around the growing void is enough to drive continued expansion. The phenomenon has been studied by Hill and coauthors $[1,2]$ for spherically symmetric conditions, and the instabilities also occur for spherical voids subject to more general axisymmetric conditions as long as the remote hydrostatic stress is much higher than the remote effective Mises stress $[3,4]$. The phenomenon has also been studied for nonlinear elasticity (Ball [5], Horgan and Abeyarathne [6], Horgan and Poligone [7]) where a cavitation instability has been interpreted as the growth of a pre-existing void, or as a bifurcation from a homogeneously stressed solid with no void to a solid containing a void.

Sufficiently high stress levels are typically reached in metal-ceramic systems, where the constraint on plastic flow in the metal leads to very high stress triaxialities. For a thin metal layer bonding two ceramic blocks Dalgleish et al. [8] have observed that under tension normal to the layer the constrained plastic flow tends to promote rapid void growth. Also, for a ceramic reinforced by Al particles a single dominant void has been observed on some of the particles crossed by a fracture surface (Flinn et al. [9]), and similar observations have been made in model experiments for a ductile particle bridging a crack (Ashby et al. [10]).

As the relevant stress states are near pure hydrostatic tension it is to be expected that initially spherical voids remain close to spherical during the huge cavity growth, as is found in the analyses. To test the influence of non-spherical initial void shapes Tvergaard and Hutchinson [11] have carried out axisymmetric analyses for a unit cell containing a single very small void with an initially spheroidal shape. As in previous analyses of cavitation instabilities the material was here represented by standard J2 flow theory. Both prolate and oblate spheroidal voids were considered, and it was found in all cases that the voids rapidly approached a spherical shape during void growth, so that the current shapes were rather independent of the initial shape when the void volume had increased by a factor around 50 .

The influence of plastic anisotropy on unstable cavity growth has been analysed by the authors [12], using a quasidynamic method to obtain an approximate solution for full $3 \mathrm{D}$, in cases where the cavities are initially spherical. This method did lead to good estimates for the critical stress, but the end shape of the voids could not be determined, as rather strong oscillations of the void surface points remained, while extremely small displacement rates were prescribed at the outer surfaces of the unit cell. Here a quasi-static analysis is carried out, using a procedure very similar to that applied for larger void volume fractions relevant to studies of ductile fracture in structural components [13].

Here, the effect of the initial cavity shape and the evolution of the shape is studied in more detail. It is shown that for an anisotropic material the void shape develops towards a spheroidal shape, which is very different for different anisotropies.

\section{Problem Formulation}

Unstable growth of 3D spheroidal voids in an infinite medium is to be investigated numerically. Fig. 1 shows the geometry with the dimensions and coordinate axes used. This geometry is similar to the one used by the authors previously in a study of void growth for rather large void volume fractions [13].

The reference Cartesian coordinate system is denoted by 


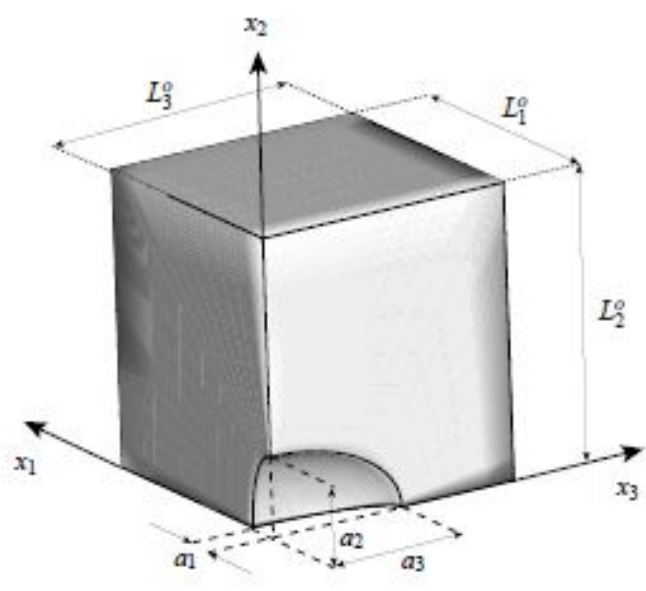

Fig. 1. Geometry of spheroidal voids analyzed. Illustrated for $w_{1}^{o}=$ $a_{2} / a_{1}=2$ and $w_{3}^{o}=a_{2} / a_{3}=\frac{1}{2}$ as well as $L_{1}^{o}=L_{2}^{o}=L_{3}^{o}$.

$x_{i}$. As shown in Fig. $1 x_{i}$ is aligned with the sides of the domain analyzed. Also the principal axes of anisotropy, $\hat{x}_{i}$, are initially aligned with the sides in every material point (not shown). No shear loads are to be applied and then the symmetries of the problem are maintained throughout the load history. Utilizing this, only one-eight of the void needs to be analyzed. The general 3D spheroidal void shape is defined by the three initial half axes, $a_{1}, a_{2}$ and $a_{3}$, from which the initial aspect ratios for the void shape are given as $w_{1}^{o}=a_{2} / a_{1}$ and $w_{3}^{o}=a_{2} / a_{3}$. An infinite medium is approximated by considering the relatively large finite initial dimensions $L_{1}^{o}=L_{2}^{o}=L_{3}^{o} \gg\left(a_{1}, a_{2}, a_{3}\right)$.

Denoting the time derivative by a superposed dot, i.e. $\frac{\partial(\bullet)}{\partial t}=(\bullet)$, the boundary conditions are expressed in terms of the displacement rates, $\dot{u}_{i}$, and the surface traction rates, $\dot{T}_{i}$, as

$$
\begin{array}{lllll}
\dot{u}_{1}=0 & ; & \dot{T}_{2}=T_{3}=0 & \text { at } & x_{1}=0 \\
\dot{u}_{2}=0 & ; & \dot{T}_{1}=\dot{T}_{3}=0 & \text { at } & x_{2}=0 \\
\dot{u}_{3}=0 & ; & \dot{T}_{1}=\dot{T}_{2}=0 & \text { at } & x_{3}=0 \\
\dot{u}_{1}=\Delta_{1} & ; & \dot{T}_{2}=\dot{T}_{3}=0 & \text { at } & x_{1}=L_{1} \\
\dot{u}_{2}=\Delta_{2} & ; & \dot{T}_{1}=\dot{T}_{3}=0 & \text { at } & x_{2}=L_{2} \\
\dot{u}_{3}=\Delta_{3} & ; & \dot{T}_{1}=\dot{T}_{2}=0 & \text { at } & x_{3}=L_{3}
\end{array}
$$

where $\Delta_{1}, \Delta_{2}$ and $\Delta_{3}$ are prescribed displacement rates used to introduce the load on the updated geometry given by the current quantaties $L_{1}, L_{2}$ and $L_{3}$. The load is characterized by the three average true normal stress components, $\Sigma_{1}, \Sigma_{2}$ and $\Sigma_{3}$, acting in the three coordinate directions, $x_{i}$. Taking $x_{2}$ as the primary loading direction, the stress components are related such that $\Sigma_{1}=\kappa_{1} \Sigma_{2}$ and $\Sigma_{3}=\kappa_{3} \Sigma_{2}$, where $\kappa_{1}$ and $\kappa_{3}$ are prescribed ratios. The stress triaxiality, $T$, is

$$
T=\frac{\Sigma_{h}}{\Sigma_{e}}=\frac{\Sigma_{1}+\Sigma_{2}+\Sigma_{3}}{3 \Sigma_{e}}=\frac{\left(\kappa_{1}+1+\kappa_{3}\right) \Sigma_{2}}{3 \Sigma_{e}}
$$

with the effective stress taken as $\Sigma_{e}=\max \left(\left|\Sigma_{2}-\Sigma_{1}\right|, \mid \Sigma_{1}-\right.$ $\left.\Sigma_{3}|,| \Sigma_{2}-\Sigma_{3} \mid\right)$.

Here, the elasto-plastically anisotropic material model used accounts for small elastic but finite plastic deformations in a visco-plastic formulation. The components of the second-order non-symmetric velocity gradient tensor, $L_{i j} \neq$ $L_{j i}$, are determined by $L_{i j}=\frac{\partial \dot{u}_{i}}{\partial x_{j}}=\dot{u}_{i},{ }_{j}$, where the velocity field components for the material are $\dot{u}_{i}$. The symmetric part of $L_{i j}$ is the strain rate, $D_{i j}=D_{j i}$, and the antisymmetric part is the continuum spin tensor, $\omega_{i j}=-\omega_{j i}$. The kinematics can then be written as [14]

$$
D_{i j}=D_{i j}^{\mathrm{e}}+D_{i j}^{\mathrm{p}} ; \quad L_{i j}=D_{i j}+\omega_{i j}
$$

where the superscripts e and p denote the elastic and plastic parts, respectively.

The elasticity relations are assumed to be given by Hooke's law. The objective rate with respect to $\omega_{i j},\left(^{\nabla}\right)$, of the symmetric Kirchhoff stress, $\tau_{i j}=\tau_{j i}$, is introduced as [14]

$$
\stackrel{\nabla}{\tau}_{i j}=C_{i j k l} D_{k l}^{\mathrm{e}}=C_{i j k l}\left(D_{k l}-D_{k l}^{\mathrm{p}}\right)
$$

Here, $C_{i j k l}$ are the isotropic elastic moduli determined by Young's modulus, $E$, and Poisson's ratio, $v$. The plastic part of the strain rate is

$$
D_{i j}^{\mathrm{p}}=\dot{\phi} N_{i j}^{\mathrm{p}} \quad ; \quad \dot{\phi}=\dot{\varepsilon}_{0}\left(\frac{J}{g}\right)^{1 / m} \quad ; \quad N_{i j}^{\mathrm{p}}=\frac{\partial J}{\partial \sigma_{i j}}
$$

Here, $J$ is the value of the anisotropic yield function to be specified later and $N_{i j}^{\mathrm{p}}=N_{j i}^{\mathrm{p}}$ is the normal to the yield surface, which gives the direction of the plastic strain increment. The magnitude of the plastic strain increment is $\dot{\phi}, \dot{\varepsilon}_{0}$ is a reference strain rate, $m$ is a strain rate sensitivity parameter and $g=g\left(\varepsilon^{\mathrm{p}}\right)=\sigma_{0}\left(1+\frac{\varepsilon^{\mathrm{p}}}{\varepsilon_{0}}\right)^{n}$ is an isotropic deformation dependent power-law hardening function. Here, $\sigma_{0}$ is the initial uniaxial reference stress in the $x_{1}$ direction, $\varepsilon_{0}=\sigma_{0} / E$, $n$ is the hardening exponent and the accumulated effective plastic strain, $\varepsilon^{\mathrm{p}}$, is

$$
\varepsilon^{\mathrm{p}}=\int \dot{\varepsilon}^{\mathrm{p}} \mathrm{d} t \quad ; \quad \dot{\varepsilon}^{\mathrm{p}}=\dot{\phi} \sqrt{\frac{2}{3} N_{i j}^{\mathrm{p}} N_{i j}^{\mathrm{p}}}
$$

The classical quadratic yield function proposed by Hill $[2,15]$ is adopted here. Referring the stresses to the principal axes of anisotropy, $\hat{x}_{i}$, defined by the orthonormal basis, $\mathbf{n}_{i}$, such that $\hat{\sigma}_{i j}=\mathbf{n}_{i} \cdot \sigma \cdot \mathbf{n}_{j}$ for $(i, j)=1,2,3$, the anisotropic yield function is

$$
\begin{aligned}
J\left(\sigma, \mathbf{n}_{i}\right)= & \sqrt{\frac{3}{2(F+G+H)}}\left[F\left(\hat{\sigma}_{22}-\hat{\sigma}_{33}\right)^{2}+G\left(\hat{\sigma}_{33}-\hat{\sigma}_{11}\right)^{2}+\right. \\
& \left.H\left(\hat{\sigma}_{11}-\hat{\sigma}_{22}\right)^{2}+2 N \hat{\sigma}_{12}^{2}+2 L \hat{\sigma}_{23}^{2}+2 M \hat{\sigma}_{13}^{2}\right]^{1 / 2}
\end{aligned}
$$


The criterion is valid for six non-zero stress components. Anisotropic hardening can be accounted for by allowing the coefficients of anisotropy, $F, G, H, N, L$ and $M$, to change with the deformations [16]. However, here the coefficients of anisotropy are assumed to remain constant. Isotropic results will also be presented, i.e. where $F=G=H=1$ and $L=M=N=3$. The final form of the constitutive relations can then be written as [14]

$$
\dot{\sigma}_{i j}=\stackrel{\nabla}{\tau}_{i j}+\omega_{i k} \sigma_{k j}+\sigma_{i k} \omega_{j k}-\sigma_{i j} D_{k k}
$$

It is noted, that $D_{k k}=D_{k k}^{\mathrm{e}} \simeq 0$ due to plastic incompressibility, where $D_{k k}^{\mathrm{p}} \equiv 0$.

\section{Numerical Method}

An updated Lagrangian formulation [14, 17] based on the principle of virtual work is used. Disregarding body forces the incremental form of the principle of virtual work in terms of the Kirchhoff stress is $[17,18]$

$$
\begin{array}{r}
\Delta t \int_{V}\left({ }_{\tau_{i j}}^{\nabla} \delta D_{i j}-\sigma_{i j}\left(2 D_{i k} \delta D_{k j}-L_{k j} \delta L_{k i}\right)\right) \mathrm{d} V= \\
\Delta t \int_{S} \dot{T}_{i} \delta \dot{u}_{i} \mathrm{~d} S-\left[\int_{V} \sigma_{i j} \delta D_{i j} \mathrm{~d} V-\int_{S} T_{i} \delta \dot{u}_{i} \mathrm{~d} S\right]
\end{array}
$$

where $V$ is the volume and $S$ is the surface, $T_{i}=\sigma_{i j} n_{j}$ are the tractions and $\delta \dot{u}_{i}$ are the virtual velocities, all referred to the current deformed configuration. Therefore, $\tau_{i j}$ is identical to $\sigma_{i j}$. The bracketed terms in Eq. (9) vanish if the current state satisfies equilibrium. However, due to numerical errors the solution tends to drift away from the true equilibrium path, and including the bracketed terms in Eq. (9) as an additional load term, prevents such drifting.

For the numerical finite element solution the cell is discretisized using iso-parametric, quadratic 20-node brick elements with three translational degrees of freedom per node. Reduced $2 \times 2 \times 2$ Gauss integration is adopted within each element. Fig. 2 shows an example of a mesh consisting of 1500 elements (21588 DOFs) for a cell given by $w_{1}^{o}=0.75$ and $w_{3}^{o}=1$ as well as $L_{1}^{o}=L_{2}^{o}=L_{3}^{o}$. The elements near the void are intentionally made rather elongated, in order to compensate for the large deformations expected during void expansion. In each increment, the time step, $\Delta t$, for the next increment is adjusted according to $\left(\dot{\varepsilon}^{\mathrm{p}}\right)_{\max } \cdot \Delta t \leq 10^{-5}$, where $\left(\dot{\varepsilon}^{\mathrm{p}}\right)_{\max }$ is the maximum effective plastic strain rate in any Gauss point.

At the point of unstable void growth the applied external load will not increase and is then not an appropriate quantity to prescribe. Instead, the combined finite element and Rayleigh-Ritz procedure as proposed by [19] is used to enforce continuous void growth. In addition, the procedure is also used to maintain the specified stress ratios, $\kappa_{1}$ and $\kappa_{3}$.
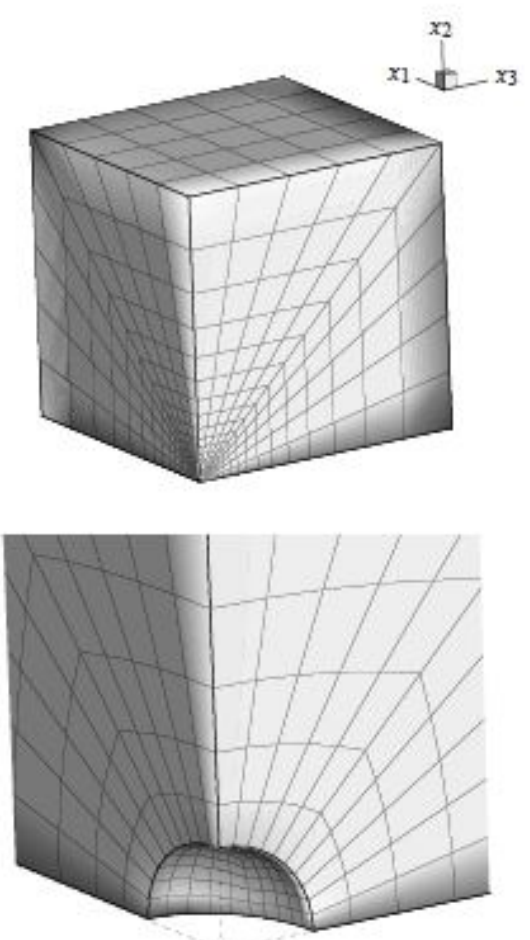

Fig. 2. Example of mesh for spheroidal void $\left(w_{1}^{o}=0.75\right.$ and $w_{3}^{o}=$ 1 with $L_{1}^{o}=L_{2}^{o}=L_{3}^{o}$ ) using 1500 elements. A zoom of the mesh near the void is also shown.

\section{Results}

Most of the numerical analyses are carried out for the material parameters $\sigma_{0} / E=0.002, v=1 / 3, \dot{\varepsilon}_{0}=0.001 \mathrm{~s}^{-1}$, $\mathrm{m}=0.01$ and $n=0.1$. Initially, a spherical void is considered with the initial half axes $a_{1}=a_{2}=a_{3}$ so small that the initial void volume fraction in the unit cell is $f_{0}=5 \cdot 10^{-7}$. The unit cell is cubic with the initial lengths of the cell sides $L_{1}^{o}=$ $L_{2}^{o}=L_{3}^{o}$. Also, in the first studies the material is taken to be an isotropic Mises solid, i.e. where $F=G=H=1$ and $L=$ $M=N=3$ in Eq. (7), to be able to compare with previous results. As expected, the voids here remain nearly spherical during growth, so that $w_{1} \simeq 1$ and $w_{3} \simeq 1$.

In Fig. 3 the stress-ratios are specified by $\kappa_{1}=\kappa_{3}=0.9$, and the curves show the macroscopic stress versus the void volume, given by the normalized values $\Sigma_{2} / \sigma_{0}$ and $V / V_{0}$. The stress is calculated as

$$
\Sigma_{2}=\frac{1}{L_{1} L_{3}} \int_{S} T_{2} \mathrm{~d} S=\frac{1}{L_{1} L_{3}} \int_{0}^{L_{1}} \int_{0}^{L_{3}}\left[T_{2}\right]_{x_{2}=L_{2}} \mathrm{~d} x_{1} \mathrm{~d} x_{3}
$$

where $S$ is the surface in the current deformed geometry. The normalized current void volume is found by numerical integration of the void volume divided by the initial volume of the void, $V / V_{0}$. In the present analyses void growth is followed up to rather large increases, $V / V_{0}=60$, but in other studies using remeshing values of $V / V_{0}$ as large as $10^{9}$ have been reached [20]. The curve for $\sigma_{0} / E=0.002$ and $m=0.01$ shows a plateau level of $\Sigma_{2} / \sigma_{0} \simeq 6.51$. Curves are also 


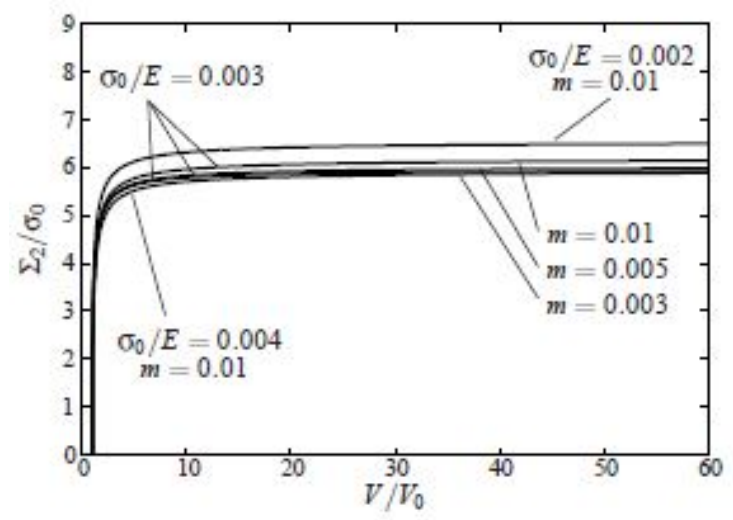

Fig. 3. Normalized stress in the primary load direction versus current normalized void volume for spherical voids, $w_{1}^{o}=w_{3}^{o}=1$, loaded by $\kappa_{1}=\kappa_{3}=0.9(T=28 / 3)$. Effects of rate sensitivity, $m$, and initial yield stress, $\sigma_{0} / E$, for isotropic plasticity are shown.
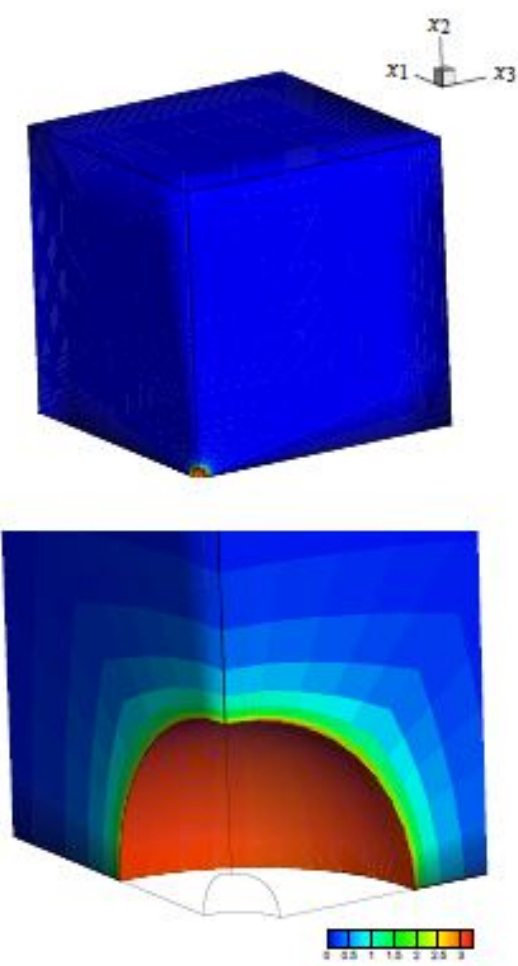

Fig. 4. Contours of accumulated effective plastic strain, $\varepsilon^{\mathrm{p}}$, at $V / V_{0}=60$ for the isotropic reference case with $\sigma_{0} / E=0.002$ and $m=0.01$ in Fig. 3 .

shown for $\sigma_{0} / E=0.003$ and $\sigma_{0} / E=0.004$, with the corresponding plateau levels 6.16 and 5.91. This agrees with the finding in [3] that the critical stress for cavitation decreases for increasing value of the yield strain. The critical stress values found here for the elastic-viscoplastic material are a little higher than those found for a time independent elasticplastic material, as was also observed in [12]. To study this further, the analysis for $\sigma_{0} / E=0.003$ and $m=0.01$ has been repeated in Fig. 3 for two smaller values of the strain-rate sensitivity exponent, $m=0.005$ and $m=0.003$, which illustrates that the predicted critical stress is reduced as the material becomes less viscous. The smallest value of $m$ gives the critical stress level 5.93, slightly smaller than the value found in [12], but still higher than the value 5.67 found for the time-independent material in [4]. All subsequent results are shown for $m=0.01$ with $\sigma_{0} / E=0.002$.

Fig. 4 shows contours of the accumulated effective plastic strain, $\varepsilon^{\mathrm{p}}$, at $V / V_{0}=60$ (an expansion of the initial radius by nearly a factor of four) for the isotropic reference case with $\sigma_{0} / E=0.002$ and $m=0.01$ in Fig. 3. Both the full domain as well as a zoom near the void are depicted. The initial and the current highly deformed geometry are both shown. As expected, a symmetric distribution of the plastic strain near the void is found. Also, the gradient of the plastic strain is very high near the void surface, i.e. through the inner-most four elements.

Two of the plastic anisotropies studied in [12] are also considered here. They are both defined by $H=1.00$ and $L=M=N=9.60$ in Eq. (7), corresponding to the experiments by Moen et al. [21] on aluminum alloy Al-7108-T7. Furthermore, $F=0.40$ and $G=7.33$ for the anisotropy labelled II, while $F=2.50$ and $G=0.30$ for the anisotropy labelled IV. These values of $F$ and $G$ are slightly more extreme compared to the experiments by Moen et al. [21], which gave $F=0.70$ and $G=3.33$. Fig. 5 illustrates the corresponding initial yield surfaces in the $\left(\sigma_{11}, \sigma_{22}\right)$-plane for $\sigma_{33}=\sigma_{12}=\sigma_{13}=\sigma_{23}=0$. Comparing to the isotropic Mises yield surface, it is seen, that while anisotropy II expands the elastic region anisotropy IV shrinks it.

Fig. 6 shows results computed for both anisotropic ma-

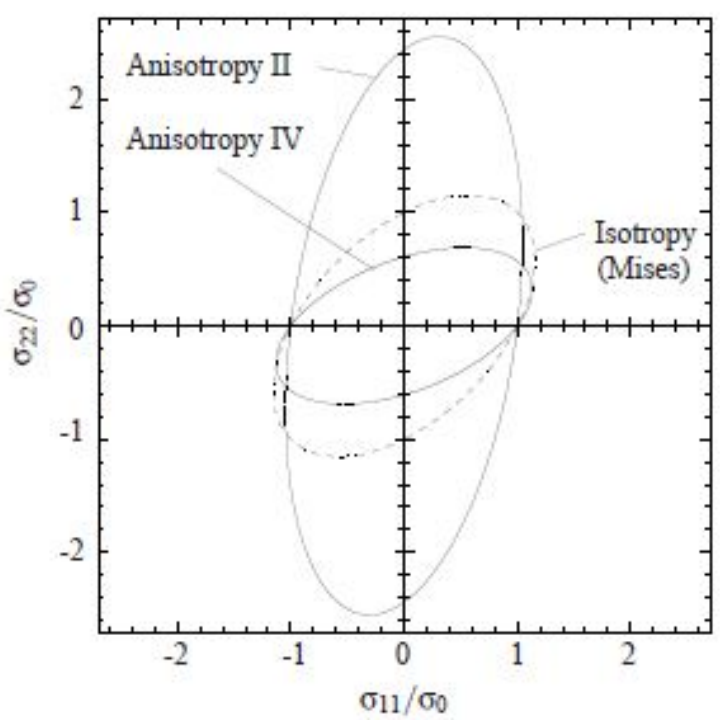

Fig. 5. Initial yield surfaces shown in the $\left(\sigma_{11}, \sigma_{22}\right)$-plane for $\sigma_{33}=\sigma_{12}=\sigma_{13}=\sigma_{23}=0$. Two different anisotropic Hill yield surfaces are shown: (II) $F=0.40$ and $G=7.33$ (IV) $F=2.50$ and $G=0.30$, while both have $H=1.00, N=L=M=9.60$ in Eq. (7). The isotropic Mises yield surface is also shown. 

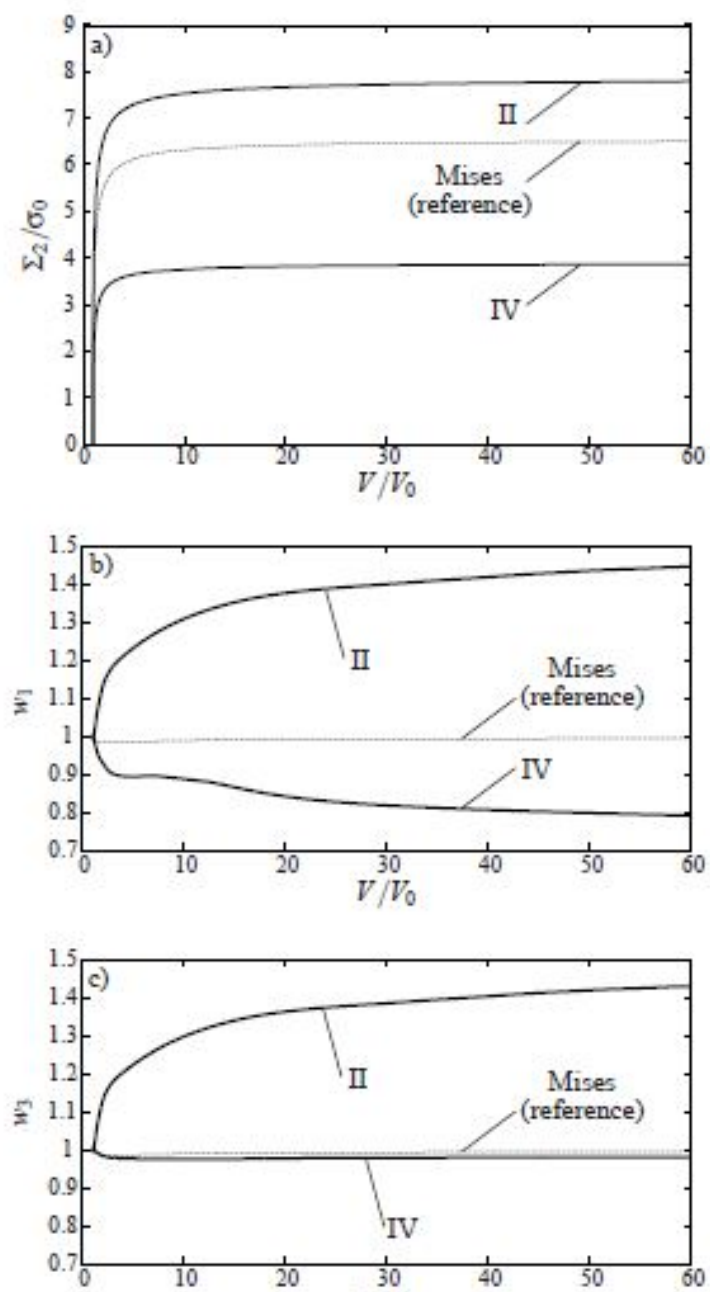

Fig. 6. Effect of plastic anisotrbpy for initially spherical voids, $w_{1}^{o}=$ $w_{3}^{o}=1.0$, loaded in pure hydrostatic tension, $\kappa_{1}=\kappa_{3}=1.0$. Isotropic reference curve with $\kappa_{1}=\kappa_{3}=0.9$ is shown for comparison. All curves have $\sigma_{0} / E=0.002$ and $m=0.01$. (a) Normalized stress in the primary load direction versus current normalized void volume. (b) Evolution of $w_{1}$. (c) Evolution of $w_{3}$.

terials, II and IV, in cases where the unit cell is subjected to pure hydrostatic tension, $\kappa_{1}=\kappa_{3}=1.0$, and where the initial void geometry is spherical, $w_{1}^{o}=1$ and $w_{3}^{o}=1$. Fig. 6a shows that anisotropy II gives a rather high critical stress, 7.80 , while anisotropy IV gives a lower critical stress, 3.87 . Figs. $6 \mathrm{~b}$ and $6 \mathrm{c}$ show that although the void shapes are spherical from the start, the shapes deviate considerably during the growth. For anisotropy II both $w_{1}$ and $w_{3}$ grow, reaching the values $w_{1}=1.45$ and $w_{3}=1.43$. Thus, the void elongates in the $x_{2}$ direction, relative to the $x_{1}$ direction and the $x_{3}$ direction. For anisotropy IV the ratio of axes $w_{1}$ decays, reaching $w_{1}=0.77$, while $w_{3}$ changes only little, to $w_{3}=0.98$. This means that the void elongates in the $x_{1}$ direction, relative to the other two directions. Apparently, both anisotropies make the initially spherical void develop into a spheroidal shape, which is approximately axisymmetric, but where the longest axis is in the $x_{2}$ direction for anisotropy II, while the longest axis is in the $x_{1}$ direction for anisotropy IV. Fig. 5 shows that anisotropy II has a lower initial yield stress in the $x_{1}$ direction than that of the $x_{2}$ direction, i.e. the material is most stiff in the direction of the longest void axis. Similarly, Fig. 5 shows that anisotropy IV has a lower initial yield stress in the $x_{2}$ direction than that of the $x_{1}$ direction, i.e. the material is again most stiff in the direction of the longest void axis.

In all cases the mesh becomes rather distorted in the radial direction, where the surface elements become very thin. For the isotropic case, Fig. 4, the deformations are rather uniform along the surface of the void, but for the anisotropic cases the distortion is more pronounced at the tip of the deformed spheroidal void, where also the circumferential distortion becomes rather large.
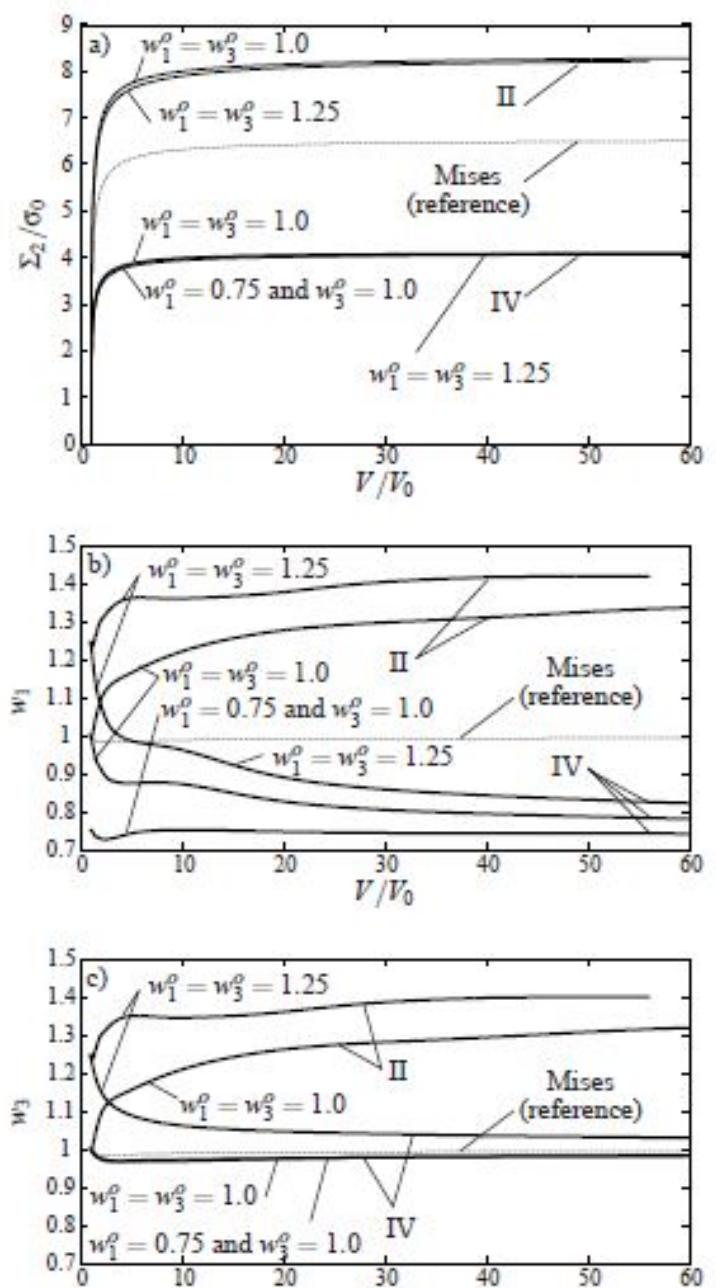

Fig. 7. Effect of initial void shape, $w_{1}^{\text {To }} /$ and $w_{3}^{o}$, and plastic anisotropy when loaded by $\kappa_{1}=\kappa_{3}=0.9$. Isotropic reference curve is shown for comparison. All curves have $\sigma_{0} / E=0.002$ and $m=0.01$. (a) Normalized stress in the primary load direction versus current normalized void volume. (b) Evolution of $w_{1}$. (c) Evolution of $w_{3}$.

As a reference case in Fig. 6 is used the curves for $\sigma_{0} / E=0.002$ in Fig. 3, which corresponds to the different external stress state $\kappa_{1}=\kappa_{3}=0.9$. This case is used as 
reference in all the subsequent figures. Here, for the Mises material, Figs. $6 \mathrm{~b}$ and $6 \mathrm{c}$ show that the void remains nearly spherical, as was also mentioned in relation to Fig. 3.

The curves in Fig. 7 are obtained for the stress state characterized by $\kappa_{1}=\kappa_{3}=0.9$, as in Fig. 3. Cases with initially spherical voids are studied for both anisotropies, but also different initially spheroidal voids are considered to obtain a better understanding of the effect of the void shape. For anisotropy II the initially spherical void leads to the stress level $\Sigma_{2} / \sigma_{0}=8.29$ and the shape parameters $w_{1}=1.34$ and $w_{3}=1.33$. A spheroidal void, $w_{1}^{o}=w_{3}^{o}=1.25$, with an initial shape rather similar to the expected final shape, leads to nearly the same critical stress, 8.22 , and a somewhat more deformed void with $w_{1}=1.42$ and $w_{3}=1.40$.

For anisotropy IV in Fig. 7 three different initial void shapes are considered, all resulting in essentially the same value of the critical stress, $\Sigma_{2} / \sigma_{0}=4.09$. For the initially spherical void the shape develops to a nearly axisymmetric spheroidal void, elongated in the $x_{1}$ direction, with $w_{1}=0.77$ and $w_{3}=0.99$. Also a spheroidal void, $w_{1}^{o}=0.75$ and $w_{3}^{o}=1.0$, with an initial shape rather similar to the expected final shape, leading to nearly the same shape of the much larger void after growth, $w_{1}=0.74$ and $w_{3}=0.99$. In addition, a computation was carried out with the initial shape also considered for anisotropy II, $w_{1}^{o}=w_{3}^{o}=1.25$. In this case the void shape parameters quickly change towards the values found in the other cases with anisotropy IV, reaching the values $w_{1}=0.83$ and $w_{3}=1.03$. It is noticed that the final void shapes found in Fig. 7 are rather similar to those found in Fig. 6 for a different applied stress state, when comparing the same anisotropies.

In Fig. 8 different applied stress states are considered for anisotropy IV, mostly with an initially spherical void. The stress states are still in the range of high stress triaxiality, where a cavitation instability limit will be reached. For $\kappa_{1}=$ 0.9 and $\kappa_{3}=0.95$ the critical stress found is $\Sigma_{2} / \sigma_{0}=4.04$ and the shape develops to $w_{1}=0.76$ and $w_{3}=0.99$. These values are very close to those found in Fig. 7 for $\kappa_{1}=\kappa_{3}=$ 0.9 . For $\kappa_{1}=0.9$ and $\kappa_{3}=1.0$ the critical stress found is $\Sigma_{2} / \sigma_{0}=3.97$ and the shape develops to essentially the same, $w_{1}=0.76$ and $w_{3}=0.99$. When $\kappa_{1}=0.85$ and $\kappa_{3}=0.95$ is considered, the critical stress found is $\Sigma_{2} / \sigma_{0}=4.07$ and the shape develops to $w_{1}=0.75$ and $w_{3}=0.99$.

In Fig. 8 one computation is added that starts from a non-spherical yoid, $w_{1}^{o}=0.75$ and $w_{3}^{o}=1.0$, with stresses specified by $\kappa_{1}=0.85$ and $\kappa_{3}=0.95$. Here, the critical stress found is $\Sigma_{2} / \sigma_{0}=4.06$ and the shape ends up being nearly the same as the start, $w_{1}=0.72$ and $w_{3}=0.99$. Thus, from the curves in Fig. 8 and those in Figs. 7 and 6 for anisotropy IV it may be concluded that the critical stress varies only little with differences in the external applied stress state, when the stress state is in the high triaxiality range leading to a cavitation instability. In all cases the rapidly growing void reaches a shape with the shape parameters in the vicinity of $w_{1}=0.75$ and $w_{3}=1$. The critical stress level for anisotropy IV is much lower than that in an isotropic Mises material, but also the final void shapes are very different from those in the Mises material, where the
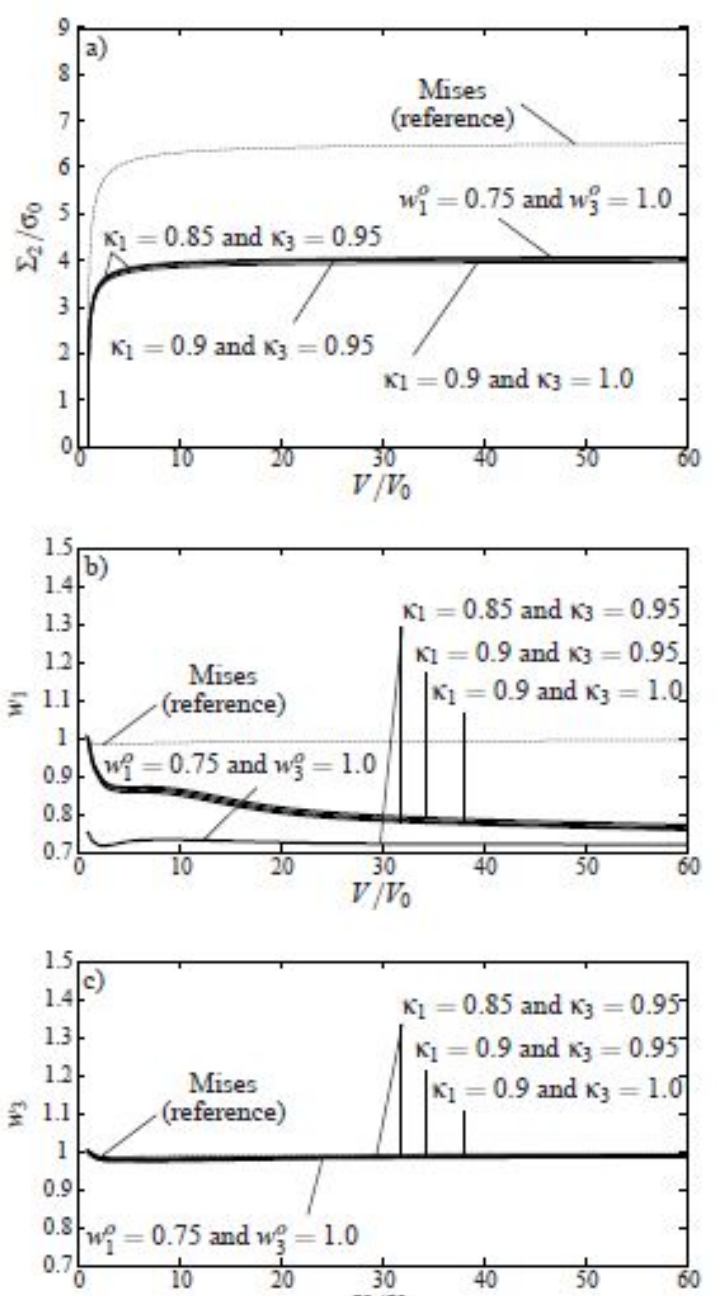

Fig. 8. Effect of loading, $\kappa_{1}$ and $\mathrm{K}_{3} V_{\text {for }}$, plastic anisotropy IV and initially spherical voids, $w_{1}^{o}=w_{3}^{o}=1$, unless else is stated. Isotropic reference curve is shown for comparison. All curves have $\sigma_{0} / E=$ 0.002 and $m=0.01$. (a) Normalized stress in the primary load direction versus current normalized void volume. (b) Evolution of $w_{1}$. (c) Evolution of $w_{3}$.

voids stay nearly spherical.

Fig. 9 shows results for anisotropy II and different initial void shapes. Three cases have initially spherical voids, for $\kappa_{1}=0.9$ and $\kappa_{3}=1.0$ the critical stress is $\Sigma_{2} / \sigma_{0}=7.87$ and the shape becomes $w_{1}=1.34$ and $w_{3}=1.33$, for $\kappa_{1}=0.9$ and $\kappa_{3}=0.95$ the critical stress is $\Sigma_{2} / \sigma_{0}=8.14$ and the shape becomes $w_{1}=1.36$ and $w_{3}=1.35$, and for $\kappa_{1}=0.85$ and $\kappa_{3}=0.95$ the critical stress is $\Sigma_{2} / \sigma_{0}=8.20$ while the shape becomes $w_{1}=1.34$ and $w_{3}=1.32$. For one of the stress states, $\kappa_{1}=0.9$ and $\kappa_{3}=0.95$, two other initial void shapes have been considered. The initial shape $w_{1}^{o}=w_{3}^{o}=$ 1.25 , which is somewhat similar to the expected final shape, results in the critical stress $\Sigma_{2} / \sigma_{0}=8.07$ and the shape becomes $w_{1}=1.45$ and $w_{3}=1.43$. On the other hand, the initial shape $w_{1}^{o}=0.75$ and $w_{3}^{o}=1.0$ is rather similar to the expected final shape for the other anisotropy, but still results in the critical stress $\Sigma_{2} / \sigma_{0}=8.16$ and the shape given by 

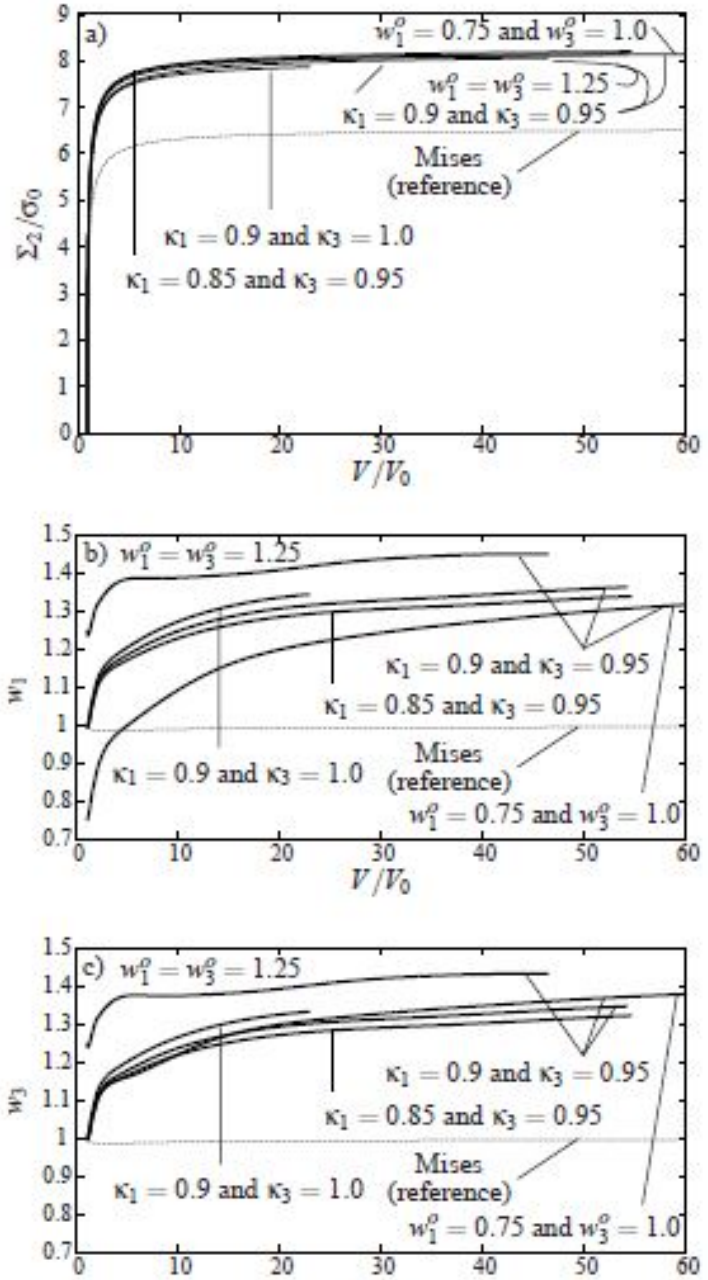

Fig. 9. Effect of loading, $\kappa_{1}$ and $/ \kappa_{3}$, and initial void shape, $w_{1}^{o}$ and $w_{3}^{o}$, for plastic anisotropy II. Isotropic reference curve is shown for comparison. All curves have $\sigma_{0} / E=0.002$ and $m=0.01$. (a) Normalized stress in the primary load direction versus current normalized void volume. (b) Evolution of $w_{1}$. (c) Evolution of $w_{3}$.

$w_{1}=1.33$ and $w_{3}=1.39$.

\section{Discussion}

Cavitation instabilities occur for a single void, far from other voids in an elastic-plastic solid, if the stress level is high enough. Then the applied outer stresses and strains reach fixed critical values, while the void keeps growing, driven by the stored elastic energy in the solid. In the present studies this behaviour is approximated by using an elasticviscoplastic analysis, and it is seen that this leads to small increases of the critical stress levels, relative to levels found in the absence of viscosity. But the general trends are well represented.

A previous 3D study by the authors, for initially spherical voids, has used a quasi dynamic method with explicit time integration and a lumped mass matrix, to avoid inverting a large stiffness matrix many times. However, this method did not make it possible to study the shape of the voids during the rapid growth when the critical stress level was reached. This is of interest since earlier axisymmetric studies for isotropic plasticity have shown that even for different spheroidal shapes of the initial void the final void shape developed to be nearly spherical.

For each of the two anisotropies considered here it is found that an initially spherical void develops into a spheroidal void that differs significantly from the initial spherical shape. For anisotropy II the values of $w_{1}$ and $w_{3}$ remain almost identical and reach values around 1.3 to 1.4 . For anisotropy IV the value of $w_{1}$ reaches values near 0.8 , while the value of $w_{3}$ remains near 1.0. These final shape values are not strongly affected by differences in the applied macroscopic stress state, in the range of high triaxialities considered here to be relevant for cavitation instabilities.

Also the effect of starting from non-spherical voids has been studied, either starting from shapes rather similar to the expected final shapes, or starting from shapes very different from the expected final shapes. In all these cases it is found that the shapes develop towards something very similar to the shapes found when starting from spherical voids.

The critical stress levels for the occurrence of cavitation instabilities are found to be much higher for anisotropy II than for anisotropy IV, with the results for isotropic plasticity in between. These stress levels are only slightly affected by the initial shape of the void, in the range of shapes considered here. The critical stress levels found in the previous analyses based on a quasi dynamic method are not directly comparable with those those obtained here, because the previous analyses used $\sigma_{0} / E=0.004$, which gives slightly lower stress levels, as seen in Fig. 3. But apart from this, there is good agreement with the critical stress levels found previously for the two anisotropies and for isotropic plasticity.

\section{References}

[1] Bishop, R. F., Hill, R., and Mott, N. F., 1945. "The theory of indentation and hardness tests". Proceedings of the Physical Society of London, 57(321), pp. 147159.

[2] Hill, R., 1950. The Mathematical Theory of Plasticity. The Clarendon Press, Oxford.

[3] Huang, Y., Hutchinson, J. W., and Tvergaard, V., 1991. "Cavitation instabilities in elastic plastic solids". Journal of the Mechanics and Physics of Solids, 39(2), pp. 223-241.

[4] Tvergaard, V., Huang, Y., and Hutchinson, J. W., 1992. "Cavitation instabilities in a power hardening elasticplastic solid". European Journal of Mechanics Asolids, 11(2), pp. 215-231.

[5] Ball, J. M., 1982. "Discontinuous equilibrium solutions and cavitation in nonlinear elasticity". Philosophical Transactions of the Royal Society of London a (mathematical and Physical Sciences), 306(1496), pp. 557 611.

[6] Horgan, C. O., and Abeyaratne, R., 1986. "A bifurcation problem for a compressible nonlinearly elastic 
medium - growth of a micro-void". Journal of Elasticity, 16(2), pp. 189-200.

[7] Horgan, C. O., and Polignone, D. A., 1995. "Cavitation in nonlinearly elastic solids: A review". Applied Mechanics Reviews, 48(8), p. 471.

[8] Dalgleish, B. J., Trumble, K. P., and Evans, A. G., 1989. "The strength and fracture of alumina bonded with aluminum-alloys". Acta Metallurgica, 37(7), pp. 1923-1931.

[9] Flinn, B. D., Rühle, M., and Evans, A. G., 1989. "Toughening in composites of $\mathrm{Al}_{2} \mathrm{O}_{3}$ reinforced with Al”. Acta Metallurgica, 37(11), pp. 3001-3006.

[10] Ashby, M. F., Blunt, F. J., and Bannister, M., 1989. "Flow characteristics of highly constrained metal wires". Acta Metallurgica, 37(7), pp. 1847-1857.

[11] Tvergaard, V., and Hutchinson, J. W., 1993. "Effect of initial void shape on the occurrence of cavitation instabilities in elastic-plastic solids". Journal of Applied Mechanics-transactions of the Asme, 60(4), pp. 807812.

[12] Legarth, B. N., and Tvergaard, V., 2010. "3D analyses of cavitation instabilities accounting for plastic anisotropy". Zeitschrift Fuer Angewandte Mathematik Und Mechanik, 90(9), pp. 701-709.

[13] Legarth, B. N., and Tvergaard, V., 2018. "Effects of plastic anisotropy and void shape on full threedimensional void growth". Journal of Applied Mechanics, 85(5), p. 051007.

[14] Yamada, Y., and Sasaki, M., 1995. "Elastic-plastic large deformation analysis program and lamina compression test". International Journal of Mechanical Sciences, 37(7), pp. 691-707.

[15] Hill, R., 1948. "A theory of the yielding and plastic flow of anisotropic metals". Proceedings of the Royal Society of London, A193, pp. 281-297.

[16] Legarth, B. N., 2008. "Necking of anisotropic microfilms with strain-gradient effects". Acta Mechanica Sinica, 24(5), pp. 557-567.

[17] McMeeking, R. M., and Rice, J. R., 1975. "Finiteelement formulations for problems of large elasticplastic deformation". International Journal of Solids and Structures, 11, pp. 601-616.

[18] Legarth, B. N, 2007. Strain-gradient effects in anisotropic materials". Modelling and Simulation in Materials Science and Engineering, 15, pp. S71-S81.

[19] Tvergaard, V., 1976. "Effect of thickness inhomogeneities in internally pressurized elastic-plastic spherical shells". Journal of the Mechanics and Physics of Solids, 24, pp. 291-304.

[20] Tvergaard, V., 1997. "Studies of void growth in a thin ductile layer between ceramics". Computational Mechanics, 20(1-2), pp. 186-191.

[21] Moen, L. A., Langseth, M., and Hopperstad, O., 1998. "Elastoplastic buckling of anisotropic aluminum, plate elements". Journal of Structural Engineering, pp. 712719 . 